\title{
Multiple Parameters Estimation of BOC Signal in Multipath Environment
}

\author{
Yang Wang*, Yang Zhou \\ Chongqing Key Laboratory of Wireless Communications, Chongqing University of Posts and Telecommunications, Chongqing 400065, \\ China
}

\begin{abstract}
For the multiple parameters estimation of BOC signal at the low SNR and multipath environment, the method which applies the characteristics of cyclic spectrum is developed. Firstly, the cyclic spectrum function of multipath BOC signal is derived. Then the cyclostationary property of BOC signal is analyzed. Finally, a novel parameter estimation algorithm of BOC signal, which makes use of the position of cyclic spectral peaks to estimate the carrier frequency, the subcarrier rate and PN code rate, is present. In the experiment the time-domain smoothing method is used to calculate the cyclic spectrum of BOC signal when the frequency is equal to zero in order to avoid the multidimensional search. At the same time using the method of the cumulative average can increase the SNR margin of the algorithm. Simulation results show that the multiple parameters of multipath BOC signal can be estimated effectively and the estimation performance is associated with the multipath environment.
\end{abstract}

\section{Introduction}

BOC signal has been widely used in various navigation and communication systems, such as Galileo, GPS navigation system and Chinese Beidou navigation system, because its good spectral splitting characteristics can effectively solve the interference of signals in the common bands. BOC modulation technology has the potential to become a mainstream technology in the various navigation systems and spread spectrum communication, so characteristic analysis for BOC signal will be very significance. The current study of BOC signal mostly was concentrated in the synchronization [1] and capture [2-3]. At the same time the characteristics of the signal are also part of the study[4]. However the study for the parameters estimation of BOC signal is less. The parameter estimation is completed by the relationship between the peak of auto-correlation function and the spreading code and sub-carrier rate of BOC signal, but the performance is not satisfactory[5]. DS signal is a second order cyclostationary signal, where the information of chip width, carrier frequency and amplitude of signal is included in the amplitude of cyclic spectrum. A novel parameter estimation algorithm of DS signal,which make use of the position and magnitude of non-zero cyclic spectral peaks to estimate the three parameters, is present[6]. The cyclostationary property of multipath DS signals is analyzed, the expressions of both the cyclic correlation function and cyclic spectrum are deduced, and a novel parameter estimation algorithm of DS signals is presented based on cyclic spectrum amplitude[7]. Obviously BOC signal is also a second order cyclostationary signal, so in this paper, for BOC signal at the low SNR and multipath environment, the blind estimation of parameters is completed by its cyclostationary characteristics.

This paper is organized as follows. Firstly ,spectral correlation function ${ }^{[8-10]}$ of multipath $\mathrm{BOC}$ signal is deduced, and then analyze the specific steps of the algorithm. Finally, the effectiveness and performance are verified by simulation experiments.

\section{Signal model}

In multipath environment, BOC signal can be expressed as

$$
r(t)=\sum_{i=1}^{M} A_{i} s\left(t-\tau_{i}\right)+n(t)
$$

where, $s(t)$ is the BOC signal and where $M, A_{i}, \tau_{i}$ denote the number of multipath components and the amplitude attenuation factor and delay of the $i$ th multipath component. $n(t)$ is the channel noise, which we assume to be white. BOC signal $s(t)$ expression is:

$$
s(t)=\sum_{n=-\infty}^{\infty} a_{n} q_{T_{c}}\left(t-n T_{c}-t_{0}\right) \cos \left(2 \pi f_{0} t+\phi_{0}\right)
$$

where $q_{T_{c}}(t)$ represents a pseudo-code chip which is multiplied with the subcarrier:

$$
q_{T_{c}}(t)=\sum_{l=0}^{N-1}(-1)^{m} u_{T_{s}}\left(t-l T_{s}\right)
$$

\footnotetext{
* Corresponding author: wangyang@ @cqupt.edu.cn
} 
where $a_{n} \in\{-1,+1\}$ is the spreading code with data modulate and where $t_{0}, \phi_{0}, N$ denote the delay, early phase and modulation order. $u_{T_{s}}(t)$ is normalized chip waveform of duration $T_{s}\left(N T_{s}=T_{c}\right) . R_{c}=1 / T_{c}$, $R_{s}=1 /\left(2 T_{s}\right)$ are the pseudo code rate and subcarrier rate.

\section{Algorithm principle}

\subsection{The cyclic spectrum function of multipath}

\section{BOC signal}

Firstly, the cycle auto-correlation function of the multipath BOC signal can be calculated by the formula (1)(3)

$$
\begin{aligned}
& R_{r}^{\alpha}(\tau)=\sum_{m=1}^{M} A_{m}^{2} R_{s}^{\alpha}(\tau) e^{-j 2 \pi \alpha \tau_{m}}+ \\
& \sum_{m=1}^{M} \sum_{n \neq m} A_{m} A_{n} R_{s}^{\alpha}\left(\tau-\tau_{m}+\tau_{n}\right) e^{-j \pi \alpha\left(\tau_{m}-\tau_{n}\right)}
\end{aligned}
$$

where $R_{s}^{\alpha}(\tau)$ is the cyclic auto-correlation function of BOC signal.We can get the cyclic spectrum function by formula(6) with Fourier transform.

$$
\begin{aligned}
S_{r}^{\alpha}(f) & =\int_{-\infty}^{+\infty} R_{r}^{\alpha}(\tau) e^{-j 2 \pi f \tau} d \tau \sum_{m=1}^{M} A_{m}^{2}\left|\int_{-\infty}^{+\infty} R_{s}^{\alpha}(\tau) e^{-j 2 \pi f \tau} d \tau\right| e^{-j 2 \pi \alpha \tau_{m}}+\sum_{m=1}^{M} \sum_{n \neq m} A_{m} A_{n}\left|\int_{-\infty}^{+\infty} R_{s}^{\alpha}\left(\tau-\tau_{m}+\tau_{n}\right) e^{-j 2 \pi f \tau} d \tau\right| e^{-j 2 \pi \alpha\left(\tau_{m}-\tau_{n}\right)} \\
& =\sum_{m=1}^{M} A_{m}^{2}\left|\int_{-\infty}^{+\infty} R_{s}^{\alpha}(\tau) e^{-j 2 \pi f \tau} d \tau\right| e^{-j 2 \pi \alpha \tau_{m}}+\sum_{m=1}^{M} \sum_{n \neq m} A_{m} A_{n}\left|\int_{-\infty}^{+\infty} R_{s}^{\alpha}\left(\tau-\tau_{m}+\tau_{n}\right) e^{-j 2 \pi f\left(\tau-\tau_{m}-\tau_{n}\right)} d \tau\right| e^{-j 2 \pi f\left(\tau_{m}-\tau_{n}\right)} e^{-j \pi \alpha\left(\tau_{m}-\tau_{n}\right)} \\
& =\sum_{m=1}^{M} A_{m}^{2} S_{s}^{\alpha}(f) e^{-j 2 \pi \alpha \tau_{m}}+\sum_{m=1}^{M} \sum_{n \neq m} A_{m} A_{n} S_{s}^{\alpha}(f) e^{-j 2 \pi(\alpha / 2+f)\left(\tau_{m}-\tau_{n}\right)}
\end{aligned}
$$

where, $S_{s}^{\alpha}(f)$ is the cyclic spectrum function of BOC

signal:

$$
S_{s}^{\alpha}(f)=\left\{\begin{array}{lr}
\frac{1}{4 T_{c}}\left[Q\left(f+f_{0}+\alpha / 2\right) Q^{*}\left(f+f_{0}-\alpha / 2\right)+Q\left(f-f_{0}+\alpha / 2\right) Q^{*}\left(f-f_{0}-\alpha / 2\right)\right], & \alpha=k / T_{c} \\
\frac{1}{4 T_{c}}\left[Q\left(f-f_{0}+\alpha / 2\right) Q^{*}\left(f+f_{0}-\alpha / 2\right)\right], & \alpha=2 f_{0}+k / T_{c} \\
\frac{1}{4 T_{c}}\left[Q\left(f+f_{0}+\alpha / 2\right) Q^{*}\left(f-f_{0}-\alpha / 2\right)\right], & \alpha=-2 f_{0}+k / T_{c}
\end{array}\right.
$$

where,

$$
Q(f)=\int_{-\infty}^{+\infty} q_{T_{c}}(t) e^{-j 2 \pi f t} d t
$$

$q_{T_{c}}(t)$ is the rectangular pulse sequence with $\{ \pm 1\}$ alternating. For BOC $(10,5)$, there is

$$
Q(f)=-\mathrm{j} \cdot \frac{\sin \left(4 \pi f T_{s}\right) \sin \left(\pi f T_{s}\right)}{\pi f \cos \left(\pi f T_{s}\right)}
$$

The cyclic spectrum of multipath BOC signal can be calculated by formula(5)(6).

When $\alpha=k / T_{c}$ ( $k$ is an integer):

$$
S_{r}^{\alpha}(f)=\sum_{m=1}^{M} A_{m}^{2} \frac{1}{4 T_{c}}\left[\begin{array}{l}
Q\left(f+f_{0}+\frac{\alpha}{2}\right) Q^{*}\left(f+f_{0}-\frac{\alpha}{2}\right)+ \\
Q\left(f-f_{0}+\frac{\alpha}{2}\right) Q^{*}\left(f-f_{0}-\frac{\alpha}{2}\right)
\end{array}\right] e^{-j 2 \pi \alpha_{0}} e^{-2 \pi \alpha \tau_{m}}+\sum_{m=1}^{M} \sum_{n \neq m} A_{m} A_{n} \frac{1}{4 T_{c}}\left[\begin{array}{l}
Q\left(f+f_{0}+\frac{\alpha}{2}\right) Q^{*}\left(f+f_{0}-\frac{\alpha}{2}\right)+ \\
Q\left(f-f_{0}+\frac{\alpha}{2}\right) Q^{*}\left(f-f_{0}-\frac{\alpha}{2}\right)
\end{array}\right]^{-j 2 \pi \alpha t_{0}} e^{-j 2 \pi(\alpha / 2+f)\left(\tau_{m}-\tau_{n}\right)}
$$

When $\alpha= \pm 2 f_{0}+k / T_{c}$ ( $k$ is an integer):

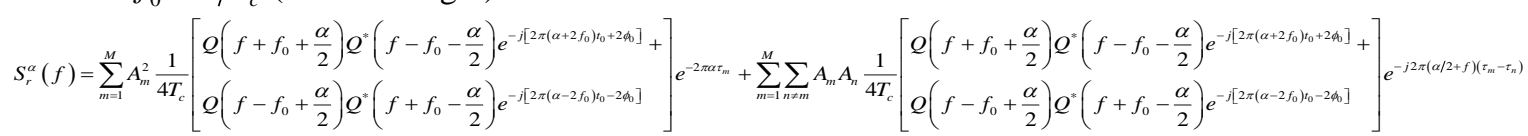

\subsection{Multipath BOC signal parameter estimation principle}

$$
\left|S_{x}^{\alpha}(f=0)\right|=\left\{\begin{array}{lr}
\frac{1}{4 T_{c}}\left|Q\left(f_{0}+\alpha / 2\right) Q^{*}\left(f_{0}-\alpha / 2\right)+Q\left(-f_{0}+\alpha / 2\right) Q^{*}\left(-f_{0}-\alpha / 2\right)\right| \sum_{m=1}^{M} A_{m}^{2} e^{-j 2 \pi \alpha \tau_{m}}+\sum_{m=1}^{M} \sum_{n \neq m} A_{m} A_{n} e^{-j \pi \alpha\left(\tau_{m}-\tau_{n}\right)} \mid, \alpha=k / T_{c} \\
\frac{1}{4 T_{c}}\left|Q\left(-f_{0}+\alpha / 2\right) Q^{*}\left(f_{0}-\alpha / 2\right)\right| \sum_{m=1}^{M} A_{m}^{2} e^{-j 2 \pi \alpha \tau_{m}}+\sum_{m=1}^{M} \sum_{n \neq m} A_{m} A_{n} e^{-j \pi \alpha\left(\tau_{m}-\tau_{n}\right)} \mid, & \alpha=2 f_{0}+k / T_{c} \\
\frac{1}{4 T_{c}}\left|Q\left(f_{0}+\alpha / 2\right) Q^{*}\left(-f_{0}-\alpha / 2\right)\right| \sum_{m=1}^{M} A_{m}^{2} e^{-j 2 \pi \alpha \tau_{m}}+\sum_{m=1}^{M} \sum_{n \neq m} A_{m} A_{n} e^{-j \pi \alpha\left(\tau_{m}-\tau_{n}\right)} \mid, & \alpha=-2 f_{0}+k / T_{c}
\end{array}\right.
$$

Analysis of the formula(11) shows that the envelope of BOC signal in multipath environment is similar to one in the single-path environment when $f=0$, just more than a product parameter, the size of the peak depends on the parameters. We can make 


$$
\rho=\left|\sum_{m=1}^{M} A_{m}^{2} e^{-j 2 \pi \alpha \tau_{m}}+\sum_{m=1}^{M} \sum_{n \neq m} A_{m} A_{n} e^{-j \pi \alpha\left(\tau_{m}-\tau_{n}\right)}\right|
$$

So formula(11)can be expressed as

$$
\left|S_{x}^{\alpha}(f=0)\right|=\left\{\begin{array}{lr}
\frac{\rho}{4 T_{c}} \mid Q\left(f_{0}+\alpha / 2\right) Q^{*}\left(f_{0}-\alpha / 2\right)+ & \alpha=k / T_{c} \\
Q\left(-f_{0}+\alpha / 2\right) Q^{*}\left(-f_{0}-\alpha / 2\right), & \rho \rho \\
\frac{\rho}{4 T_{c}}\left|Q\left(-f_{0}+\alpha / 2\right) Q^{*}\left(f_{0}-\alpha / 2\right)\right|, & \alpha=2 f_{0}+k / T_{c} \\
\frac{\rho}{4 T_{c}}\left|Q\left(f_{0}+\alpha / 2\right) Q^{*}\left(-f_{0}-\alpha / 2\right)\right|, & \alpha=-2 f_{0}+k / T_{c} \\
0, & \text { else }
\end{array}\right.
$$

To facilitate a clearer understanding of the circulation characteristics of its envelope, from formula(8)(13) and $T_{c}=4 T_{s}$ for BOC $(10,5)$, we can get

When $\alpha=k / T_{c}$ :

$$
\left|S_{x}^{\alpha}(f=0)\right|=8 \rho T_{s}\left|\begin{array}{l}
\frac{\sin \left(4 \pi T_{s} f_{0}-\pi k / 2\right) \sin \left(4 \pi T_{s} f_{0}+\pi k / 2\right)}{\pi^{2}\left(64 T_{s}^{2} f_{0}^{2}-k^{2}\right)} \\
\tan \left(\pi T_{s} f_{0}-\pi k / 8\right) \tan \left(\pi T_{s} f_{0}+\pi k / 8\right)
\end{array}\right|
$$

When $\alpha= \pm 2 f_{0}+k / T_{c}$ :

$$
\left|S_{x}^{\alpha}(f=0)\right|=4 \rho T_{s} \frac{\sin ^{2}(\pi k / 2) \tan ^{2}(\pi k / 8)}{\pi^{2} k^{2}}
$$

From the analysis of Fig.2, we can see that the cyclic spectrum section with $f=0$ is symmetrical about zero frequency. There are four main peaks whose positions are located in $+2 f_{0}+1 / T_{s}, \quad+2 f_{0}-1 / T_{s}$, $-2 f_{0}+1 / T_{s},-2 f_{0}-1 / T_{s}$. At the same time there are four maximum sub-peaks whose positions are located in $+2 f_{0}+1 / T_{s}-1 / T_{c} \quad, \quad+2 f_{0}-1 / T_{s}+1 / T_{c}$ $-2 f_{0}+1 / T_{s}-1 / T_{c},-2 f_{0}-1 / T_{s}+1 / T_{c}$. Therefore, the carrier frequency and subcarrier rate can be estimated by the interval between the main peak in the positive 1 axis and one in the negative axis, and the pseudo-code rate can be estimated by the interval between the main peak and the maximum sub-peak next to the main peak.

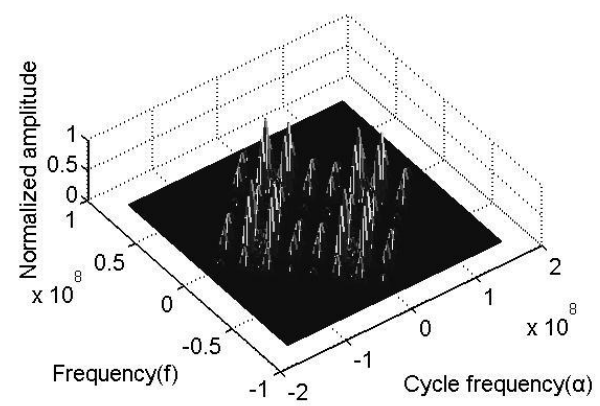

Fig 1. the cyclic spectrum of multipath BOC signal

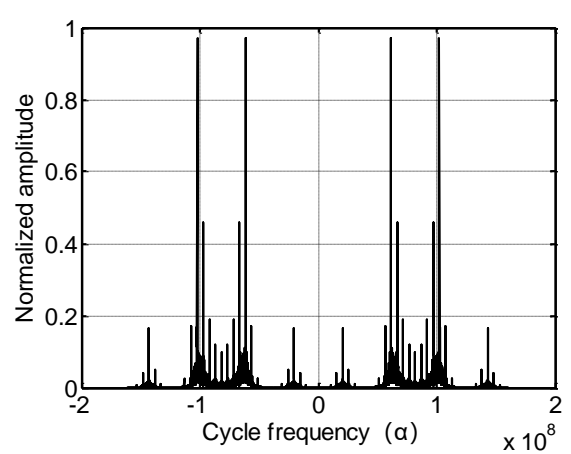

Fig 2. the cyclic spectrum section of multipath BOC signal $f=0$

\subsection{Parameter estimation algorithm}

In this paper, the time smoothing algorithm is adoped, and only calculate the $f=0$ section of the multipath BOC signal, to reduce the computational complexity.

Specific steps parameter estimates can be obtained by the above principle:

(1) Segment the sampled signal according to a certain length (ensure that the length contains multiple pseudo-code cycle), so $M$ groups of data vector can be obtained.

(2) The absolute value $S_{i}$ of the cyclic spectrum section at $f=0$ for i-th group data $(i \in[1, M])$ can be calculated.

(3) Accumulate and average the cyclic spectrum section to reduce the noise effect,then the averaged result $\bar{S}=\frac{1}{M} \sum_{i=1}^{M} S_{i}$ can be obtained.

(4) Search the two main peaks whose positions are located in $n_{1}, n_{2}\left(n_{2}>n_{1}\right)$ in the positive axle of $\bar{S}$, then the carrier frequency and subcarrier rate can be evaluated by $f_{0}=\left(n_{1}+n_{2}\right) \cdot \Delta f / 4 \quad$ and $R_{s}=\left(n_{2}-n_{1}\right) \cdot \Delta f / 4 . \Delta f=1 /(Z-1) T_{\Delta}$ is the spectral resolution.

(5) Search the two maximum sub-peaks whose positions are located in $n_{3}, n_{4}\left(n_{4}>n_{3}\right)$ between the two main peaks, then the pseudo code rate can be evaluated by $R_{c}=\left(n_{3}-n_{1}\right) \cdot \Delta f$.

\section{Simulations}

In order to verify the effectiveness and performance of the algorithm, make the following simulation. Experimental parameters: BOC $(10,5)$ signal with sine subcarrier, carrier frequency $f_{0}=40.92 \mathrm{MHz}$, the pseudo code rate $R_{c}=5.115 \mathrm{MHz}$, the pseudo code cycle $N_{c}=63 b i t$, the subcarrier rate $R_{s}=10.23 \mathrm{MHz}$, the sampling frequency $f_{s}=163.68 \mathrm{MHz}$. Data length is 4096. Define the Rhine factor $Q$ which means the ratio between the power of line-of-sight component and the 
sum power of all the scattered wave. And when $Q \rightarrow 0$, the channel is approximately degenerate for Rayleigh channel. Use NRMSE(Normalized Root Mean Square Error) to measure the estimation performance of the algorithm.

Experiment one: Compare the algorithm performance of the parameter estimation for BOC signal in the single-path and multipath environment. The cumulative number is ten.

Analyzed from the Fig.3, Fig.4 and Fig.5 in the single-path and multipath environment, NRMSE of the carrier frequency, the subcarrier rate and pseudo code rate are changed with the SNR. Their NRMSE all become bigger when the SNR is lower. Since there is no interference of multipath components in the single path channel, the estimation performance is best. While in the Rayleigh channel( $Q=0.01)$, the main dominant signal is not present, the estimation performance is worst. And in the Les channel $Q=10$, the main dominant signal is present, so its parameter estimation performance is better than one in the Rayleigh channel. However because of the multipath effects, its estimation performance is worse than one in the single-path channel. At the same time by comparative analysis among the Figs.4, Figs.5 and Figs.6, the estimation performance of the carrier frequency is the best, the estimation performance of subcarrier rate rank second, and they are better than the performance of the pseudo code rate. This is due to the estimations of the carrier frequency and the subcarrier rate are based on the relative position between the main peaks, while the estimations of the pseudo-code rate is based on the relative position between the main peak and the maximum sub-peaks. Since the absolute value of sub-peak is lower, it is more easily affected by noise.

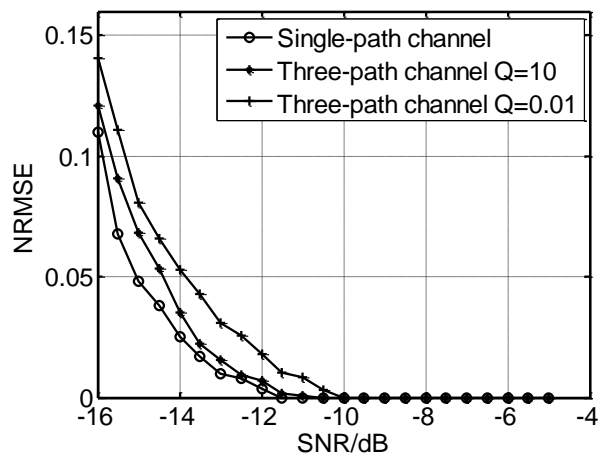

Fig 3. Estimation performance of the carrier frequency in different channels

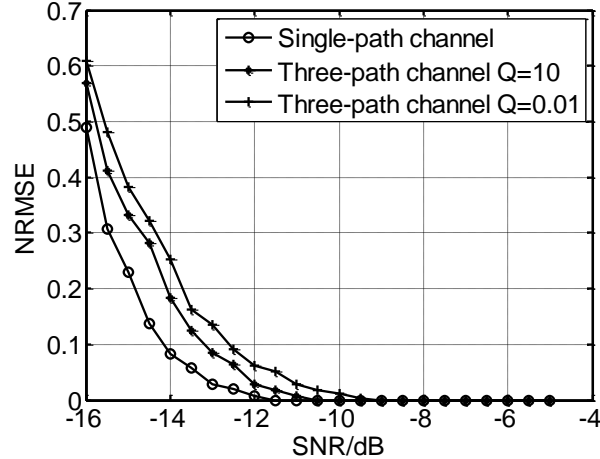

Fig 4. Estimation performance of the subcarrier rate in different channels

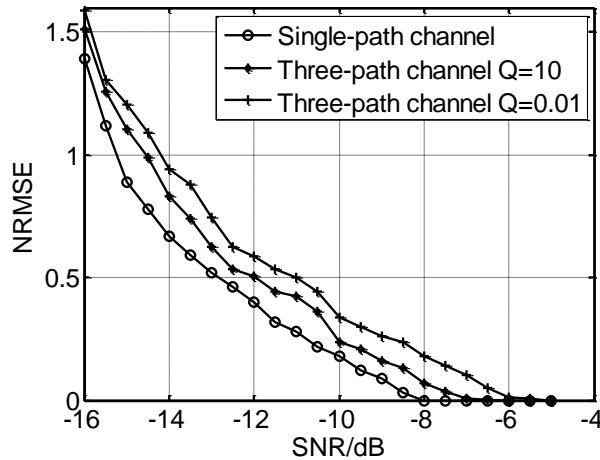

Fig 5. Estimation performance of the pseudo code rate in different channels

\section{Conclusion}

For most of the wireless communication channel with the multipath environment, the cyclic spectrum function of multipath BOC signal is derived. Then the characteristic of the section of cyclic spectrum envelope is analyzed to realize the multiple parameters estimation. Simulation results show that this method can effectively achieve the estimation of the carrier frequency, the subcarrier rate and the pseudo-code rate at the low SNR and multipath environment. At the same time analyze the different performance of the algorithm between the single-path and multipath conditions, and analyze the different performance of the algorithm for the carrier frequency, subcarrier rate, the pseudo code rate. This method can realize the multiple parameters estimation of multipath BOC signal. This can lay the foundation for the fine feature extraction of BOC signal, such as the pseudo-code sequence estimation.

\section{Acknowledgements}

This work was supported by the National Natural Science Foundation of China (No. 61601073), the Research Project of Chongqing Educational Commission (No. KJ1600417) and Doctorate Start-up Program of Chongqing University of Posts and Telecommunications (A2015-28). 


\section{References}

1. Qi Jiamin, Chen Jiapin, Li Zhenbo, et al. Unambiguous BOC modulated signals synchronization technique [J]. IEEE Communications Letters, 2012, 16(7): 986-989.

2. Lee Youngpo, Chong Dahae, Song lickho, et al. Cancellation of correlation side-peaks for unambiguous BOC signal tracking [J]. IEEE Communications Letters, 2012, 16(5): 569-572.

3. F.Benedetto, G. Giunta,E. S. Lohan,et al. A Fast Unambiguous Acquisition Algorithm for BOC-Modulated Signals[J]. IEEE Transactions on Vehicular Technology, 2013, 62(3): 1350-1355.

4. Wang Manxi, Li Hong, Ma Yifei, et al. Analysis on the characteristics of $\mathrm{BOC}$ signals [J]. Aerospace Electronic Warfare, 2008, 24(3): 52-55.

5. Qian Bo, Tian Haoming, Feng Yongxin, et al. Reasearch on an Algorithm of Correlation Detection for BOC Modulation Signal [J]. Fire Control \& Command Control, 2011, 36(4):22-25.

6. Huang Chunlin, Liu Zheng, Jiang Wenli. Chip width, carrier frequency and amplitude estimation of DS signal based on cycle spectrum amplitude[J]. Acta Electronica Sinica, 2002,30(9):1353-1356.

7. Shi Jianfeng, Wang Keren. Parameter estimation of multipath direct-spread signal based on cyclic spectrum amplitude[J]. Systems Engineering and Electronics,2008,30(8):1427-1430.

8. Gardner W A. Spectral correlation of modulated signals: part I-analog modulation [J]. IEEE Transactions on Communication, 1987, 35(6): 584-594.

9. Gardner W A, Brown W, Chih-kang Chen. Spectral correlation of modulated signals: part II-Digital modulation[J]. IEEE Transactions on Communication, 1987, 35(6): 595-601.

10. CHEN C K. Spectral correlation characterization of modulated signals with application to signal detection and source location [D]. Davis: Electrical Engineering and Computer Science in the Graduate Division, University of California, 1989. 